\title{
An overview on the clinical application of radiobiological modeling in radiation therapy of
} cancer

\begin{abstract}
Analysing the dose distribution inside target volumes of cancer patients before radiation delivery and then selection of the biologically optimal dose distribution has been one of the crucial steps in recent treatment planning developments. Plan evaluation and optimization have been based on the physical dose distribution and dose-volume parameters for several decades. However, with the development of

a.clinical radiobiology in both domains of tumor and normal tissue response to radiation, ii) existence of reliable clinical results, and

b. Emergence of new mathematical models in cancer biology and treatment, radiation scientists have been motivated to calculate tumor control probability (TCP) and normal tissue complication probability (NTCP) for modern complex clinical treatment plans. The prediction of clinical outcome in terms of TCP in radiation therapy and its development have been an interesting subject of investigations for several decades. Additionally, this process has provided new information on radiotherapy consequences such as increased local control rates and lower complications rates. It also has helped treatment teams to choose optimum plans for individual patients. In this overview, we will look into some of these studies and give emphasis on potential benefits of TCP/NTCP calculations in different areas of radiation therapy such as plan evaluation, and the uncertainties associated with dose delivery.
\end{abstract}

Keywords: radiation therapy, treatment planning, radiobiological modelling, tumor control probability, normal tissue complication probability
Volume 2 Issue I - 2017

\begin{abstract}
Asghar Mesbahi, ',2 Maryam Oladghaffari' 'Medical Physics Department, Tabriz University of Medical Sciences, Iran

${ }^{2}$ Radiation Oncology Department, Tabriz University of Medical Sciences, Iran
\end{abstract}

Correspondence: Asghar Mesbahi, Associate Professor of Medical Physics, Medical Physics Department, Tabriz University of Medical Sciences, Medical School,Attar Street, Tabriz, Iran, Email amesbahi20I0@gmail.com

Received: September 2I, 2016 | Published: January 05, 2017
Abbreviations: TCP, tumor control probability; NTCP, normal tissue control probability; LKB, lyman kutcher burman; IGRT, image guided radiation therapy; IMRT, intensity modulated radiation therapy; SIB-IMRT, simultaneous integrated boost-IMRT; VMAT, volumetric modulated arc therapy; DA, double arc; SA, single arc; HT, helical tomotherapy; IFP, improved forward planning; NPC, nasopharynx cancer; EPID, electronic an imaging portal device; SIB, simultaneous integrated boost; HDR, high dose rate; HIPO, hybrid inverse treatment planning optimization; IPSA, inverse planning simulated annealing

\section{Introduction}

Recent progress in radiation therapy of cancer has been in several main branches such as new irradiation technologies and mathematical modelling of tumor and normal tissue response to ionising radiation. Application of radiobiological modelling in radiation therapy plans goes back three decades and recently it has been the investigational focus for several research centers to bring this modeling tool in clinical practice. ${ }^{1-4}$ However, there have been several shortcomings and uncertainties in radiobiological modeling such as the lack of clinical data on different cancer types as well as insufficiency of radiobiological modeling in consideration of all biological characteristics related to the tumor and normal tissue. Nonetheless, in recentyears several manufacturers have implemented Tumor Control Probability (TCP) and Normal Tissue Control Probability (NTCP) modelling on their commercial Treatment Planning Systems (TPS)s. Biological modelling basically utilizes the DVH of a given plan and biological parameters of tumor type and normal critical tissues for calculation of TCP and NTCP. In its general form, TCP for a tumor is usually calculated using the Poisson model by the following formula:

$$
T C P=\exp (-N)
$$

Where the $\mathrm{N}$ is the number of surviving clonogenic cells after irradiation.

Generally speaking, in the Poisson model of TCP the radiation response of a particular tumour is mainly described by the linear term of the linear quadratic cell survival model. TCP calculations for a population of patients with variable radiobiological parameters can be calculated by the following formula:

$$
T C P=\sum_{i} g_{i}\left(\sigma_{\alpha}\right) \cdot T C P\left(\alpha_{i}, \beta_{i}\right)
$$

Where, the TCP indicates the TCP averaged over a population with variability in radiosensitivity $\left(\alpha_{i}\right)$ which is simulated as a Gaussian distribution of $\alpha_{i}$ with $\bar{\alpha}$ mean and $\sigma_{\alpha}$ standard deviation. The $\beta_{i}$ parameter denotes the repairable part of radiation damage which varies over the nnpulation of patients. Also $g_{i}$ shows the fraction of patients having $\alpha_{i}$ as their radiosensitivity. ${ }^{4}$

There are several models for NTCP calculations using DVH, dosimetric data of each patient.[4] One of the most applied models is the Lyman-Kutcher-Burman (LKB) model. ${ }^{1}$ The NTCP calculation in the LKB model is defined as: 


$$
\begin{aligned}
& \operatorname{NTCP}(D, v)=\frac{1}{\sqrt{2 \pi}} \int_{-\infty}^{t} \exp \left(-\frac{1}{2} x^{2}\right) d x \\
& \mathrm{t}=\frac{D-T D_{50}(v)}{m \cdot T D_{50}(v)} \\
& T D_{50}(v)=\frac{T D_{50}(1)}{v^{n}}
\end{aligned}
$$

Where $\operatorname{TD}_{50}(v)$ is the tolerance dose for a $50 \%$ complication probability caused by uniform irradiation to volume $v$, and where $n$ is the volume exponent and $m$ is a parameter that is inversely related to the steepness of the dose-response curve.

The increasing number of publications on radiobiological modeling implies its progressive utilization in current clinical practice as well as in silico (computer) modelling research. Although, the main purpose of TCP/NTCP calculations has been to provide a surrogate tool for plan comparisons and optimum plan selection for a given treatment, there are many other investigations that have employed this approach to quantize the radiobiological consequences for different available modalities, and geometric errors, as well as comparing novel techniques for radiation therapy. ${ }^{2,5-24}$ Moreover, several studies have utilized radiobiological modeling to evaluate the effect of geometric errors in image guided radiation therapy (IGRT), while others have applied this modeling for brachytherapy planning as well as radiation therapy with heavy ion beams. ${ }^{25-33}$

This article intends to review briefly the recent studies on application of radiobiological modeling in current radiation therapy practice. Additionally, it is an attempt to gather the information on the application of radiobiological modeling in various areas of research in clinical practice and to represent the potential of current biological modeling in radiation therapy.

\section{Application of TCP/NTCP for plan compari- son}

In recentyears, there has been an increasing tendency in application of radiobiological modeling to evaluate and predict the clinical outcome of treatment plans. Dosimetric comparisons between three dimensional conformal therapy (3D-CRT) and intensity modulated radiation therapy (IMRT), radiobiological models have been used widely to explore the predicted biological differences between two these techniques. Biological evaluation of 3D-CRT and IMRT plans for the treatment of prostate cancer has been reported in several studies. In a study by Deb and Fielding, 3D-CRT and IMRT plans of prostate cancer were compared for the same daily and total doses. The results revealed that the IMRT plan significantly lowers the NTCP for the rectum while a small gain in TCP was found. In a similar study by Landry et al. ${ }^{23}$ a total number of 10 patients with adenocarcinoma of the pancreatic head were planned simultaneously with 3D-CRT and inverse-planned IMRT and compared. In the IMRT plans, small bowel received $25 \%$ less dose which lead to three times lower NTCP for IMRT plans. As can be noted from the above mentioned studies, the advantage of IMRT in lowering the NTCPs of small bowel and rectum was evident, because IMRT had reduced critical organ doses relative to 3D-CRT in most cases. However, TCP has very slight variation with absorbed dose in the range of 70 to $80 \mathrm{~Gy}$ for prostate cancer. Basically, this can be related to the reduction of steepness of doseresponse curve in higher doses.

Biological models have been exploited to compare new and more complex treatment protocols in localized prostate carcinoma. For example, Bansal et al. ${ }^{34}$ compared standard 3D-CRT followed by an IMRT boost with a simultaneous integrated boost-IMRT (SIBIMRT) treatment plan. ${ }^{34}$ Considering the prescribed dose of $74 \mathrm{~Gy}$ to the PTV, the volume of rectum receiving 70 Gy or more was $25 \%$ lower with SIB-IMRT compared to sequential-IMRT. Compared to sequential-IMRT, SIB-IMRT reduced the mean doses to both bladder and rectum by $13 \%$ and $17 \%$, respectively for all thirty patients. However, if precisely evaluating the NTCP results, it becomes clear that NTCP reductions are not in complete accordance with a dose reduction in the bladder and rectum. According to their results, an NTCP of $0.86 \pm 0.75 \%$ and $0.01 \pm 0.02 \%$ for the bladder was seen for sequential-IMRT and SIB-IMRT plans respectively, which means 80 times decrease in NTCP compared to $13 \%$ dose reduction. But, with SIB-IMRT technique, $17 \%$ decrease in rectum dose lead to 1.4 times lower NTCP for rectum. This example clearly exhibits a paradigm shift when a dosimetric perspective is replaced by a radiobiological metrics for treatment plan interpretation.

The comparison between these two treatment techniques has not been limited to prostate cancer . In a clinical report, radiobiological evaluations of plans for high grade glioma were carried out to compare IMRT versus 3D-CRT. ${ }^{9}$ The effect of radiation dose escalation from 59.6Gy to $90 \mathrm{~Gy}$ on TCP and NTCP in ten patients was assessed. The results revealed no difference in TCP between 3DCRT and IMRT at doses of $59.4 \mathrm{~Gy}$ and $90 \mathrm{~Gy}$. Nonetheless, dose escalation to $90 \mathrm{~Gy}$ increased TCP by approximately $25 \%$, relative to $59.4 \mathrm{~Gy}$. This study showed that IMRT of high grade glioma causes a reduction (70\%) in the normal tissue complications for this brain legion.

Radiological modeling also has been used for plan comparisons for the volumetric modulated arc therapy (VMAT) technique. In this regard, several studies aimed to compare the double arc (DA) and single arc (SA) plans of using RapidArc technique for prostate cancer. A study reported that the SA and DA techniques produced similar TCP for low-risk prostate cancer. Furthermore, in spite of better dose distributions for DA in most of the cases, the NTCP for the femoral heads and bladder were not statistically different between the SA and DA plans. However, the DA technique showed a slightly lower NTCP for rectum. ${ }^{35}$ On the other hand, in another study, in contrast to the previous study, DA plans provided a better rectal and bladder dose distribution by shifting the delivered dose from the anterior-posterior direction to the lateral. Moreover, the TCP of the prostate in the DA plan was found to be slightly higher $(0.16 \%)$ compared to the SA. The results of both studies confirmed that using DA instead of SA does not cause tremendous improvement in TCP for prostate cancer. Moreover, radiobiological studies on esophageal cancer show that SIB treatments with RapidArc leads to better results compared to a standard dose plan. Using the RapidArc technique, acceptable dose escalation was clinically achievable for most of patients with more than $18 \%$ gain in TCP and a slight acceptable increase in NTCP for both the heart and lung. ${ }^{36}$

The advantages and shortcomings of new dose delivery techniques such as IMRT and Helical tomotherapy (HT) have been discussed in terms of practical use and dosimetric benefits in the literature. 
Otherwise,_biological comparisons of helical tomotherapy with IMRT could provide more information on the effectiveness of these treatment techniques. Chatterjee et al performed radiobiological comparison between HT, forward-planned IMRT and two-phase conformal plans for radical radiotherapy of head and neck squamous cell carcinomas. Dose escalation using HT showed the radiobiological advantage in terms of TCP compared to other techniques. However, TCP for the IMRT plans was comparable to that of HT plans, whereas TCP for the two-phase technique was lower than the two other methods.

Ferreira et al. compared radiobiological effectiveness of forward and inverse IMRT using different fractionations for head and neck tumors. ${ }^{13}$ An improved forward planning (IFP) technique and two IMRT techniques using different fractionation schemes were planned. Following irradiation organ response was estimated using the relative seriality model and the Poisson Linear-Quadratic-Time model to take into account cell repopulation in the primary tumor during the fractionated treatment. An average TCP of $38 \%$ was found for conventional radiotherapy which increased to $80 \%$ with IFP, and $85 \%-89 \%$ for the two IMRT techniques. The findings revealed that the shorter treatment time and larger dose per fraction with IMRT resulted in an $11 \%$ increase in TCP. Additionally, the average NTCP was reduced from $80 \%$ with conventional to $31 \%$ with IMRT plans. The TCP and NTCP calculations showed a significant improvement in treatment outcome with IMRT compared to conventional radiation therapy.

Although most of radiobiological modeling has been applied on treatment plans to compare rival plans for one treatment case, more practical and promising applications of radiobiological modeling are being performed on IMRT plan optimization. It should be noted that more recently the application of biological modeling has been proposed and its commercial and approved software has been installed in new TPSs for IMRT optimization purposes. ${ }^{20,37}$ For instance, the Eclipse TPS (Varian Medical Systems, Ca, USA) is only capable of doing optimization on static IMRT plans and not VMAT plans. A study assessed the performance of the biologically based IMRT optimization of Eclipse compared to its dose/dose volume optimization model for nasopharynx cancer (NPC). It was found that the target coverage and conformity were comparable between the two approaches, while biologically optimized plans showed more hot spots. The NTCP of the parotid glands produced by the biological method were 3times lower than dosimetric approach for both early and advanced stage cases. It was concluded that the biologic approach could be a potential alternative to the dosimetric approach for nasopharynx IMRT planning. ${ }^{20}$

Recently, biological modeling has been used to compare photon beam treatments plans with proton therapy treatment plans. In this regard, Zhang et al compared the risks of cardiac toxicity in two pediatric patients undergoing photon or proton radiotherapy. The risks of developing cardiac toxicity after photon versus proton radiotherapies for a pediatric patient with Hodgkin disease and a pediatric patient with medulloblastoma were estimated. Their results indicated that in the Hodgkin patient, the relative risk for cardiac toxicity was about $13 \%$ higher for photon beams compared to proton beams; while the medulloblastoma patient showed a 7times higher risk for cardiac toxicity using photons. ${ }^{38}$

According to basic radiobiological studies, radiation with high linear energy transfer (LET) shows a lower dependency on tumor oxygen level in their biological damage to irradiated organs. Thus, to overcome hypoxic tumor radio-resistance, the technique of LETpainting was suggested. In the LET-painting method, high LET radiation is only directed to hypoxic compartments, while lower LET radiation is applied to normoxic tissues. In a recent experiment, Carbon-12 and oxygen-16 ion treatment plans with four fields were applied on an oropharyngeal cancer case with an identified hypoxic entity within the tumor. They found that the LET-painting concept using oxygen-16 ions on hypoxic tumors resulted in a considerable increase in TCP when applying only a slight dose boost. ${ }^{39}$

\section{Biological consequences of setup errors and organ movements}

Currently, patient repositioning before treatment and after daily setup is performed in many different external radiotherapy modalities to reduce geometric uncertainties associated with external radiation treatments. Furthermore, new on-board imaging modalities such CBCT make it possible for physicists and radiotherapists to evaluate treated anatomic site and compare planned and treated volumes. Moreover, this dosimetric evaluation provides required information for radiobiological evaluation of setup variation and their effect on planned TCP and NTCP. For example, according to Jin et al, random setup errors shift the TCP curve to the right, while systematic errors compress the TCP curve downward. ${ }^{19}$ In this part of review we will consider investigations on radiobiological consequences of geometric uncertainties in external radiation therapy.

Portal imaging devices have been employed to record geometric accuracy of treatment fields during and before treatment. ${ }^{40}$ Due to their vital role in patient daily functioning, reducing radiation damage to heart and lungs have been the major concerns in radiotherapy of breast cancer. In a radiobiological study, the setup errors and organs movement were monitored and recoded by electronic an imaging portal device (EPID)s and the NTCP of the heart calculated. In research by Louwe et al. ${ }^{41} 3 \mathrm{D}$ reconstruction of the actual heart dose during breast cancer treatment using EPID was performed for NTCP calculation. ${ }^{41}$ The results showed that EPID dosimetry can be employed for invivo verification of the heart dose and NTCP estimation in radiation therapy of breast cancer.

The radiobiological effect of tumor and organ movement can be estimated using new 4DCT imaging used for radiation therapy planning. Naturally, respiration causes unavoidable motion and deformation in normal organs and tumors located in thorax region. These variations during respiration can lead to considerable difference between planned the delivered dose and its spatial distribution in 3D treatment techniques. In a study by Ronald et al, radiobiological analyses assessed the clinical significance of the observed discrepancies between the 3D and 4DCT image-based dose distributions by planning all the phases of a 4DCT image set. The Radiobiological index of $\mathrm{P}+$ which indicates probability of complication-free tumor control probability was calculated for each plan. It was found small differences (less than 2\%) in $\mathrm{P}+$ between gating and static conventional methods, while slightly higher differences in $\mathrm{P}+(4.0 \%$ on average $)$ were seen between the tracking regimen and static conventional treatment .

Like other previous dosimetric studies the results suggested that for some patients $4 \mathrm{D}$ planning provides no extra benefits beyond that provided by $3 \mathrm{D}$ planning for static conventional or gated radiotherapy. ${ }^{28}$ In a similar approach by Valdes et al. ${ }^{42}$ TCPs were estimated for a range of alphas, betas and tumor cell densities. With a maximum dose difference of $5 \mathrm{~Gy}$ between $3 \mathrm{D}$ and $4 \mathrm{D}$ plans, the 
TCP was calculated for SBRT considering its typical fractionation schedules. Nevertheless, the difference in TCP increased by up to $5 \%$ between the $4 \mathrm{D}$ and $3 \mathrm{D}$ plans. Considering these biological modeling results, they concluded that 4D dose calculations are not required for most SBRT cases except for those with irregularly shaped target volumes. Another study by Partridge et al showed the potential gain in predicted TCP from dose escalation facilitated by moderate deep inspiration breath-hold technique. ${ }^{26}$ The results indicated that escalation of the prescription dose is possible from $64 \mathrm{~Gy}$ to a mean of $73.7 \pm 6.5 \mathrm{~Gy}$ without margin reduction and also a statistically significant increase in TCP was also found.

Nowadays treatment re-planning using weekly CBCT images for radiotherapy patients can be done during treatment course to provide more precise dose delivery and to circumvent erroneous treatment due to daily organ movement. Adaptive radiotherapy is defined as changing the radiation treatment plan delivered to a patient during a course of radiotherapy to account for temporal changes in anatomy such as tumor shrinkage, weight loss or internal motion, etc. However, biological consequences of this intervention during the treatment course could remain unclear for some of practitioners. To address this issue, the clinical impact of adaptive radiotherapy was assessed using biological modeling for bladder cancer. In the study of wright et al., various adaptive planning target volumes (PTVs) were generated from the inter-fractional bladder variation observed during the first four CBCT sessions. In addition to IMRT plans delivering 60Gy to a given PTV, simultaneous integrated boost (SIB) plans were generated. For a uniform clonogenic cell density in the whole bladder, TCP varied between $53 \%$ and $58 \%$ for the 60 Gy plans, while it was between $51 \%$ and $64 \%$ for the SIB plans. They showed that dose tracking and TCP calculation can provide additional information to standard criteria such as geometrical coverage for the selected cases. ${ }^{43}$

The radiobiological evaluation process has not been restricted to different treatment methods and modalities. In a different study, researchers examined the clinical impact of the Varian Exact couch on dose and volume coverage to targets and critical organs. The treatment couch and rail's attenuation and various configurations were taken into account in planning. The results showed that the TCP was reduced by up to $10.5 \%$ (6.3\% on average) due to couch attenuations. Furthermore, they concluded that the treatment couch and rails should be taken into account in treatment planning procedure, otherwise clinically unacceptable dose and volume coverage losses occur to the target. ${ }^{27}$ Radiobiological modeling of TCP or NTCP is based upon the volume irradiated at a particular dose, and it can be assumed that the introduction of IGRT can lead to an improvement in the TCP by increasing the dose conformity to PTV in daily treatment and decrease in NTCP by reducing the margins considered to compensate for setup uncertainty. The radiobiological impact of reduced margins and treatment techniques for prostate cancer in terms TCP/NTCP for IMRT and 3D-CRT treatment has been investigated. ${ }^{18} \mathrm{~A}$ marked decrease in NTCP for IMRT plans with narrow margins was seen and for comparable TCP values, narrow margins resulted in lower NTCP for all studied plans.

\section{Applications in brachytherapy planning}

Like other radiation therapy techniques, brachytherapy has also evolved to give more conformal and tailored doses to target volumes with less dose to surrounding normal organs. Recently used TPSs in brachytherapy are functioning based on CT or MRI images and exploit new optimization algorithms for precise and accurate treatment planning. To compare two contouring techniques, radiobiological modeling could be a suitable approach in brachytherapy treatments. Additionally, studies have shown that contouring based on MRI images with better soft tissue contrast provides better results than CT images in radiation therapy. ${ }^{44-48}$ In this regard, a radiobiological comparison was conducted to study the benefits of using MRI for treatment planning relative to CT-based planning for temporary implant prostate brachytherapy. ${ }^{11}$ The results indicate that brachytherapy planning by using CT images causes cold-spots that would influence the resultant TCP. On the other hand, MRI-based planning shows improvements in TCP by $6-10 \%$, depending on used parameters in radiobiological modeling.

In radiation therapy of prostate cancer, a course of high dose rate (HDR) interstitial brachytherapy is used as an alternative to boostIMRT in combination with external radiation therapy. However, we know that the interstitial brachytherapy is more invasive than IMRT and the advantages of brachytherapy application can be confirmed by the help of quantitative analysis and estimation biological outcome of treatment plans. In this regard, in a study by Fatyga et al., singlefraction HDR boosts was compared to biologically equivalent fractionated IMRT boosts, assuming two approaches of image guided IMRT and conventional IMRT. Comparing the resultant TCP and NTCP, the authors concluded that HDR delivery produces a significantly better therapeutic ratio than the conventional IMRT. Also, it was dosimetrically and radiobiologically comparable to or even better than the image guided IMRT delivery. ${ }^{[12]}$ In another recent study, a comparison of two different optimization algorithms in brachytherapy has been reported. In this study inverse planning simulated annealing (IPSA) and hybrid inverse treatment planning optimization (HIPO) algorithms were compared for brachytherapy plans of prostate cancer. Thirty patients plans were calculated with IPSA as well as HIPO to obtain radiobiological results. Using the dosimetric parameters of plans, TCP and NTCP were calculated with the BioSuite software. ${ }^{37}$ Results indicated that HIPO optimizations could reduce TCPs up to $10 \%-20 \%$ for all PTVs lower than $74 \mathrm{~cm}^{3}{ }^{3}{ }^{49}$

\section{Discussion}

In recent decades we have witnessed the advent of new treatment techniques and progress in cancer biology and imaging as well as computer sciences. These progressions have had great impacts on bringing the radiobiological modeling into radiation therapy treatment planning systems.

The studies on plan comparison between different techniques such as 3D-CRT and IMRT have emphasized that dosimetric metrics are not sufficient for plan comparison in complex and very similar plans. Because in some cases, large dosimetric differences do not lead to considerable TCP and NTCP differences between two treatment plans. This means that the biological properties of tumor and normal tissues change the scale of dosimetric differences between rival plans. Thus, considering TCP/NTCP results for compared plans could prevent the time consuming process of plan optimization and selection for very complex plans with a subtle discrepancy in TCP/NTCP.

Although studies show that IGRT applications has reduced the required margins for geometric uncertainties in treatment plans, TCP/ NTCP studies in IGRT plans as mentioned in the current review have indicated its slight but meaningful clinical impact. In other words, as far as we know, the main function of IGRT has been to avoid wrong 
patient positioning and geometrical miss in the range of more than $2-3 \mathrm{~mm}$ in daily positioning. Additionally, its gain when transformed to radiobiological metrics in terms of TCP or NTCP has shown to be significant. ${ }^{18,40,41}$

It is worth mentioning some important points about further widespread usage of radiobiological modeling for plan optimization in IMRT. Considering the current uncertainties associated with radiobiological modeling of treatment plans, and lack of biological information needed for an individual patient plan, it seems that performing biological optimization based on raw and unreliable biological data could result in erroneous dose evaluation, distribution and delivery in radiation therapy. This means that without knowing the exact value of radiobiological parameters for both of organs at risk and tumor, the TCP and NTCP calculations will be associated with a great uncertainty. Consequently, dose distributions based on radiobiological optimization will be compromised accordingly. It should be really appreciated that for accurate and reliable TCP/NTCP calculations for an individual plan, biological parameters such as, cell density, radio-sensitivity, hypoxic subvolume sizes and locations, repopulation rates and re-oxygenation rates are acquired. Otherwise, biological modeling can be considered as an extra aiding tool for plan validations.

\section{Conclusion}

The studies reviewed in this article reported the radiobiological superiority of VMAT and IMRT techniques over 3D-conformal techniques in different cases such as head- neck and prostate cancers. Also, the radiobiological effect of IGRT and patient positioning was evaluated in different studies. The results revealed increased TCP and reduced NTCP for IGRT techniques. Moreover, radiobiological evaluations were performed on brachytherapy plans. The results highlighted the potential of TCP/NTCP calculations as a comparison tool for different treatment techniques.

It can be concluded that the radiobiological modeling of treatment plans has helped physicians and radiation therapy physicists in better understanding of the outcome of various treatment plans in conjunction with currently used dosimetric criteria. Additionally, selecting optimum plan among available different plans and making a radiobiological comparison between newly emerged techniques can be facilitated by using of biological modeling in treatment planning software. However, it should be remembered that IMRT plan optimization based on approximate biological data could lead to inevitable complications for patients treated in radiation therapy. Thus, due to the shortage of experimental and validated radiobiological data on normal organs and different types of tumors, more radiobiological and clinical studies as well as model improvements are required for clinically usable biological plan optimization.

\section{Acknowledgements}

None.

\section{Conflict of interest}

Author declares that there is no conflict of interest.

\section{References}

1 Zaider M, Hanin L. Tumor control probability in radiation treatment. Med Phys. 2011;38(2):574-583.
2 Eisbruch A. Clinical heterogeneity and tumor control probability. Acta Oncol. 2010;49(8):1385-1387.

3 Szlag M, Slosarek K. Two-dimensional imaging of tumour control probabilities and normal tissue complication probabilities. Rep Pract Oncol Radiother. 2010;15(2):31-39.

4 Warkentin B, Stavrev P, Stavreva N, et al. A TCP-NTCP estimation module using DVHs and known radiobiological models and parameter sets. J Appl Clin Med Phys. 2004;5(1):50-63.

5 Battista JJ, Johnson C, Turnbull D, et al. Dosimetric and radiobiological consequences of computed tomography-guided adaptive strategies for intensity modulated radiation therapy of the prostate. Int J Radiat Oncol Biol Phys. 2013;87(5):874-880.

6 Carver A, Gilmore M, Riley S, et al. An analytical approach to acceptance criteria for quality assurance of Intensity Modulated Radiotherapy. Radiother Oncol. 2011;100(3):453-455.

7 Chatterjee S, Willis N, Locks SM, et al. Dosimetric and radiobiological comparison of helical tomotherapy, forward-planned intensity-modulated radiotherapy and two-phase conformal plans for radical radiotherapy treatment of head and neck squamous cell carcinomas. Br J Radiol. 2011;84(1008):1083-1090.

8 Chow JC, Jiang R. Prostate volumetric-modulated arc therapy: dosimetry and radiobiological model variation between the single-arc and double-arc technique. J Appl Clin Med Phys. 2013;14(3):4053.

9 De La Fuente HT, Ahmad AS, Vlachaki MT. Intensity modulated radiation therapy versus three dimensional conformal radiation therapy for treatment of high grade glioma: a radiobiological modeling study. $J$ Xray Sci Technol. 2010;18(4):393-402.

10 Deb P, Fielding A. Radiobiological model comparison of 3D conformal radiotherapy and IMRT plans for the treatment of prostate cancer. Australas Phys Eng Sci Med. 2009;32(2):51-61.

11 Dinkla AM, Pieters BR, Koedooder K, et al. Improved tumour control probability with MRI-based prostate brachytherapy treatment planning. Acta Oncol. 2013;52(3):658-665.

12 Fatyga M, Williamson JF, Dogan N, et al. A comparison of HDR brachytherapy and IMRT techniques for dose escalation in prostate cancer: a radiobiological modeling study. Med Phys. 2009;36(9):3995-4006.

13 Ferreira BC, do Carmo LM, Mateus J, et al. Radiobiological evaluation of forward and inverse IMRT using different fractionations for head and neck tumours. Radiat Oncol. 2010;5:57.

14 Haworth A, Williams S, Reynolds $\mathrm{H}$, et al. Validation of a radiobiological model for low-dose-rate prostate boost focal therapy treatment planning. Brachytherapy. 2013;12(6):628-636.

15 Hussein M, Aldridge S, Guerrero UT, et al. The effect of 6 and 15 MV on intensity-modulated radiation therapy prostate cancer treatment: plan evaluation, tumour control probability and normal tissue complication probability analysis, and the theoretical risk of secondary induced malignancies. Br J Radiol. 2012;85(1012):423-432.

16 Inaniwa T, Kanematsu N, Suzuki M, et al. Effects of beam interruption time on tumor control probability in single-fractionated carbon-ion radiotherapy for non-small cell lung cancer. Phys Med Biol. 2015;60(10):4105-4121.

17 Iori M, Cattaneo GM, Cagni E, et al. Dose-volume and biological-model based comparison between helical tomotherapy and (inverse-planned) IMAT for prostate tumours. Radiother Oncol. 2008;88(1):34-45.

18 Jensen I, Carl J, Lund B, et al. Radiobiological impact of reduced margins and treatment technique for prostate cancer in terms of tumor control pro- 
bability (TCP) and normal tissue complication probability (NTCP). Med Dosim. 2011;36(2):130-137.

19 Jin JY, Kong FM, Liu D, et al. A TCP model incorporating setup uncertainty and tumor cell density variation in microscopic extension to guide treatment planning. Med Phys. 2011;38(1):439-448.

20 Kan MW, Leung LH, Yu PK. The use of biologically related model (Eclipse) for the intensity-modulated radiation therapy planning of nasopharyngeal carcinomas. PLoS One. 2014;9(11):e112229.

21 King CR. LDR vs. HDR brachytherapy for localized prostate cancer: the view from radiobiological models. Brachytherapy. 2002;1(4):219-226.

22 Koontz BF, Das S, Temple K, et al. Dosimetric and radiobiologic comparison of $3 \mathrm{D}$ conformal versus intensity modulated planning techniques for prostate bed radiotherapy. Med Dosim. 2009;34(3):256-260.

23 Landry JC, Yang GY, Ting JY, et al. Treatment of pancreatic cancer tumors with intensity-modulated radiation therapy (IMRT) using the volume at risk approach (VARA): employing dose-volume histogram (DVH) and normal tissue complication probability (NTCP) to evaluate small bowel toxicity. Med Dosim. 2002;27(2):121-129.

24 Lee TF, Ting HM, Chao PJ, et al. Dosimetric advantages of generalised equivalent uniform dose-based optimisation on dose-volume objectives in intensity-modulated radiotherapy planning for bilateral breast cancer Br J Radiol. 2012;85(1019):1499-1506.

25 Mavroidis P, Su FC, Giantsoudi D, et al. Radiobiological and dosimetric analysis of daily megavoltage $\mathrm{CT}$ registration on adaptive radiotherapy with Helical Tomotherapy. Technol Cancer Res Treat. 2011;10(1):1-13.

26 Partridge M, Tree A, Brock J, et al. Improvement in tumour control probability with active breathing control and dose escalation: a modelling study. Radiother Oncol. 2009;91(3):325-329.

27 Pulliam KB, Howell RM, Followill D, et al. The clinical impact of the couch top and rails on IMRT and arc therapy. Phys Med Biol. 2011;56(23):7435-7447.

28 Roland T, Mavroidis P, Gutierrez A, et al. A radiobiological analysis of the effect of 3D versus 4D image-based planning in lung cancer radiotherapy. Phys Med Biol. 2009;54(18):5509-5523.

29 Selvaraj J, Uzan J, Baker C, et al. 4D radiobiological modelling of the interplay effect in conventionally and hypofractionated lung tumour IMRT. Br J Radiol. 2015;88(1045):20140372.

30 Siochi RA, Kim Y, Bhatia S. Tumor control probability reduction in gated radiotherapy of non-small cell lung cancers: a feasibility study. $J$ Appl Clin Med Phys. 2014;16(1):4444.

31 Song W, Battista J, Van DJ. Limitations of a convolution method for modeling geometric uncertainties in radiation therapy: the radiobiological dose-per-fraction effect. Med Phys. 2004;31(11):3034-3045.

32 Tudor GS, Rimmer YL, Nguyen TB, et al. Consideration of the likely benefit from implementation of prostate image-guided radiotherapy using current margin sizes: a radiobiological analysis. $\mathrm{Br} J$ Radiol. 2012;85(1017):1263-1271.

33 Luxton G, Hancock SL, Boyer AL. Dosimetry and radiobiologic mode comparison of IMRT and 3D conformal radiotherapy in treatment of carcinoma of the prostate. Int J Radiat Oncol Biol Phys. 2004;59(1):267-284.

34 Bansal A, Kapoor R, Singh SK, et al. Dosimetric comparison of standard three-dimensional conformal radiotherapy followed by intensity-modu- lated radiotherapy boost schedule (sequential IMRT plan) with simultaneous integrated boost-IMRT (SIB IMRT) treatment plan in patients with localized carcinoma prostate. Indian J Urol. 2012;s28(3):300-306.

35 Rana S, Cheng C. Radiobiological impact of planning techniques for prostate cancer in terms of tumor control probability and normal tissue complication probability. Ann Med Health Sci Res. 2014;4(2):167-172.

36 Warren S, Partridge M, Carrington R, et al. Radiobiological determination of dose escalation and normal tissue toxicity in definitive chemoradiation therapy for esophageal cancer. Int J Radiat Oncol Biol Phys. 2014;90(2):423-429.

37 Nahum AE, Uzan J. (Radio) biological optimization of external-beam radiotherapy. Comput Math Methods Med. 2012;2012:329214.

38 Zhang R, Howell RM, Homann K, et al. Predicted risks of radiogenic cardiac toxicity in two pediatric patients undergoing photon or proton radiotherapy. Radiat Oncol. 2013;8(1):184-188.

39 Bassler N, Toftegaard J, Luhr A, et al. LET-painting increases tumour control probability in hypoxic tumours. Acta Oncol. 2014;53(1):25-32.

40 Boughalia A, Marcie S, Fellah M, et al. Assessment and quantification of patient set-up errors in nasopharyngeal cancer patients and their biological and dosimetric impact in terms of generalized equivalent uniform dose (gEUD), tumour control probability (TCP) and normal tissue complication probability (NTCP). Br J Radiol. 2015;88(1050):20140839.

41 Louwe RJ, Wendling M, van Herk MB, et al. Three-dimensiona heart dose reconstruction to estimate normal tissue complication probability after breast irradiation using portal dosimetry. Med Phys 2007;34(4):1354-1363

42 Valdes $\mathrm{G}$, Robinson $\mathrm{C}$, Lee $\mathrm{P}$, et al. Tumor control probability and the utility of $4 \mathrm{D}$ vs $3 \mathrm{D}$ dose calculations for stereotactic body radiotherapy for lung cancer. Med Dosim. 2015;40(1):64-69.

43 Wright P, Muren LP, Hoyer M, et al. Evaluation of adaptive radiotherapy of bladder cancer by image-based tumour control probability modelling. Acta Oncol. 2010;49(7):1045-1051.

44 Dalah E, Moraru I, Paulson E, et al. Variability of target and norma structure delineation using multimodality imaging for radiation therapy of pancreatic cancer. Int J Radiat Oncol Biol Phys. 2014;89(3):633-640.

45 Metcalfe P, Liney GP, Holloway L, et al. The potential for an enhanced role for MRI in radiation-therapy treatment planning. Technol Cancer Res Treat. 2013;12(5):429-446.

46 Ahmed M, Schmidt M, Sohaib A, et al. The value of magnetic resonance imaging in target volume delineation of base of tongue tumours---a study using flexible surface coils. Radiother Oncol. 2010;94(2):161-167.

47 van Lin EN, Futterer JJ, Heijmink SW, et al. IMRT boost dose planning on dominant intraprostatic lesions: gold marker-based three-dimensional fusion of CT with dynamic contrast-enhanced and $1 \mathrm{H}$-spectroscopic MRI. Int J Radiat Oncol Biol Phys. 2006;65(1):291-303.

48 Parker CC, Damyanovich A, Haycocks T, et al. Magnetic resonance imaging in the radiation treatment planning of localized prostate cancer using intra-prostatic fiducial markers for computed tomography co-registration. Radiother Oncol. 2003;66(2):217-224.

49 Panettieri V, Smith RL, Mason NJ, et al. Comparison of IPSA and HIPO inverse planning optimization algorithms for prostate HDR brachytherapy. J Appl Clin Med Phys. 2014;15(6):5055. 